\title{
The sensitivity of infrared imaging for diagnosing Raynaud's phenomenon and for Thoracic Outlet Syndrome is dependend on the method of temperature extraction from thermal images
}

K. Ammer

Institute for Physical Medicine and Rehabilitation, Hanuschkrankenhaus, Vienna, Austria

Medical Imaging Research Group, Faculty of Advanced Technology, University pf Glamorgan, Pontypridd, UK

\section{Introduction}

It was shown in previous papers [1,2] that the method of extracting temperatures from thermal images has an impact on temperature readings. Recently, two studies were conducted to investigate the influence of definition of regions of interest on the diagnostic sensitivity of thermal images for Thoracic Outlet Syndrom [3] and Raynaud's Phenomenon [4] respectively.

\section{Thoracic Outlet Syndrome}

25 series of thermal images from patients who were tested for thermal symptoms caused by TOS were retrieved from the database of our Thermography Unit. Each series consisted of eight images which have been recorded with an AGEMA 870 Infrared Scanner. The retrived AGEMA images have been stored in CATS-format and were converted to the image format of the software package CTHERM. Further image analysis was performed with the statistics tool of CTHERM:

For the established evaluation, regions of interest were defined over the index and little finger of both hands in the following way: A polygonal measurement area was defined around the finger. The outline of this region of interest crossed the hand at the base of the finger and the background temperature was increased to the level of the isotherm surrounding the finger. The mean temperature and the number of pixels of the measurement area were recorded.

For the alternative evaluation a line was aligned to the base and the tip of the finger investigated. The mean temperature and the number of pixels of the line were recorded. The temperature difference between index and little finger was calculated for each method of evaluation.

Based on this temperature difference the following classification was made: a pathological temperature difference in one position was named "possible TOS", pathological findings in two positions were regarded as a "probable TOS" and pathological temperature readings in three or four arm position was classified as "definite TOS". In addition, TOS cases were labeled with "index" or "little finger" with respect to the colder finger.

Further statistical analysis was performed with the software package SPSS 10 , after the temperature readings from the measurement areas in all positions have been computed into one file resulting in a sample of 100 values, of which the reliability coefficient alpha and the average interclass correlation were determined. The recorded values from the regions of interest were compared by the Student-T test between the original and the alternative method of evaluation. The level for significant differences was set for 2-tailed $p=0.05$.

Thermal image series from 25 patients (24 females, 1 male) with median age of 45 (range 23 to 66) years were re-evaluated. Analysis of the temperature readings from each measurement area by the KolomogorovSmirnov-Test indicated a distribution of values that was not significantly different from a normal distribution (2tailed p-values between 0.06 and 0.32). Therefore, further analysis with the Student-T-Test was appropriate.

The size of of the measurement area of the alternative method of evaluation was about $10 \%$ of the region iof interest used with the original method. Interclass correlation coefficients between temperature readings obtained from the standard and the alternative method of evaluation were 0.887 for the temperature difference index minus little finger at the right hand side, and 0.794 for the left hand side.

The allocation to classes of TOS was significantly different between the two methods of evaluation (Chisquare Test, 2-tailed- $p=0.01)$. A higher number and more severe cases of TOS were detected with the alternative than with the original method of evaluation.

\section{Raynaud's Phenomenon}

Three methods of evaluation of temperature readings from thermal images were compared in hands of 26 subjects after cold challenge. The cold stress test- tool of the software package C-Therm was used for the calculation of Ring's Thermal-Index. Alternatively areas over single fingers were defined and gradients of single fingers were calculated by substracting the mean temperature of the dorsum from the mean temperature of finger areas (FG1=finger gradient 1). Temperature gradients for single fingers were determined in the following way: Spot temperatures were measured on the tip and over the mid of metacarpal bone of each finger. Gradients were calculated by subtracting the metacarpal temperature from the temperature of the finger tip (FG2=finger gradient 2). The mean value of the temperature gradients of all fingers of the right and the left hand were calculated.

The difference of both mean temperature gradients of fingers prior, 10 or 20 minutes past cold challenge were compared with the findings of the Thermal Index for the same periods.

Comparison of the thermal index 10 minutes and 20 minutes past cold challenge, obtained a mean decrease of the Thermal Index of $0.32 \pm 1,0$ at the later measurement. The absolute values of the mean FG1 were 0,93 to 1,28 greater than the related Thermal Index. The differences between FG2 and the thermal index were 0.1 to 1. 
However, analysing all thermal indices with non parametric tests obtained no significant differences between the indices. Single measure interclass correlations revealed values between 0.75 and 0.95 .

Using a threshold of -4.0 for a diagnostic thermal index, the highest number of cases with Raynaud's phenomenon were identified with TG2, followed by TG1 and the C-Therm derived Thermal Index .

Table 1. Cases with Raynaud's phenomenon detected by different methods of evaluation

\begin{tabular}{|c|c|c|c|}
\hline Time past cold challenge & Thermal Gradient 2 & Thermal Gradient 1 & Thermal index \\
\hline 10 minutes & 13 cases & 10 cases & 7 cases \\
\hline 20 minutes: & 9 cases & 5 cases & 3 cases \\
\hline
\end{tabular}

\section{Conclusion}

Smaller measurement areas led In both studies to a higher diagnostic sensitivity than larger regions of interest. This might be caused by a higher accurracy of infrared based temperature measurements from small areas taken from objects with a curved surface. However, precision is higher when measurement areas cover the total field of interest and are not just placed somewhere within the region of interest. As accuracy and precision of infrared based temperature measurements confer a different weight and importance for a diagnostic test and for a outcome measure[5], appropriate definition of measurement area may vary due to the intended utility of thermal imaging.

\section{REFERENCES}

[1] K. Ammer, Influence of Imaging and Object Conditions on Temperature Readings From Medical Infrared Images. Polish Journal of Environmental Studies 15 (2006): S117-S119,

[2] K.Ammer Repeatability of the Identification of Hot spots in Thermal Images. Thermology international, 16 (2006): 113

[3] K. Ammer Alternative Evaluation of Thermal Images Captured From Patients Suspected To Suffer From Thoracic Outlet Syndrome. In: Benkö I, Kovacsics I, Lovak I, eds.15 $15^{\text {th }}$ International Conference on Thermal Engineering and Thermogrammetry (THERMO), Abstracts, Merestechnikai, Automatizalas es informatikaj Tudomanyos Egyrsulet (MATE) Hotechnikai es Termogrammetriai (HT es TGM) Szakosztalya, Budapest, (2007)

[4] K. Ammer Three methods of evaluation of thermal images from patients with suspected Raynaud's phenomenon. Thermology international, 17 (2007): 157

[5] K. Ammer. Thermal imaging as outcome measure. Thermology international 16, (2006): 125 